\title{
Frequencies of Genetic Polymorphisms of the Cholesterol and Statin Metabolic Pathway Genes among Healthy South Indian Tamil Population
}

\author{
Chandrasekaran Indumathi', Annan Sudarsan Arun Kumar', Adithan Surendiran1', Steven Aibor Dkhar²* \\ 'Department of Pharmacology, Jawaharlal Institute of Postgraduate Medical Education and Research (JIPMER), Puducherry, INDIA. \\ ${ }^{2}$ Department of Clinical Pharmacology, Jawaharlal Institute of Postgraduate Medical Education and Research (JIPMER), Puducherry, INDIA.
}

\begin{abstract}
Background: Indians are genetically predisposed to Coronary Artery Disease (CAD), hence it is worth studying the effect of genetic polymorphisms which affect CAD development including Low- Density Lipoprotein Cholesterol (LDL-C) in our population. Studies have found that there is a considerable variation in the genetic makeup of Indians with distinct genetic groups having been identified-one such being Dravidian Tamil population. Aim:We aimed to determine the distribution of allele and genotype frequencies of genes associated with LDL-C lipid levels as well as those associated with statin metabolic pathway in 100 healthy South Indian Tamil volunteers. Results:The minor allele frequencies (MAFs) of the genetic polymorphisms of HMGCR rs5908, rs17238540 and rs12916 were 0.5, 3.5 and $41 \%$ respectively. The MAF of $L D L R$ rs688, CYP7A1 rs3808607, ABCB1 rs1128503, SLCO1B1 rs4149056 were 26, 42, 41.5 and $7 \%$ respectively. The frequencies of the genetic polymorphisms studied show considerable variation from other Indian ethnic groups in 5 out of the 7 genetic poly-
\end{abstract}

morphisms studied. Conclusion: Considerable variations in frequencies of genetic polymorphisms were found between Tamil population and other major ethnic populations worldwide with respect to HMGCR rs 17238540 and $L D L R$ rs688.

Key words: Genetic polymorphisms, Genotype frequency, Tamilians, Healthy volunteers, LDL-C, Statin pathway.

Correspondence :

Dr. Steven Aibor Dkhar, Senior Professor \& Head, Department of Clinical Pharmacology, Third floor-Institute Block, Jawaharlal Institute of Postgraduate Medical Education and Research, Dhanvantari Nagar, Pondichery, India

Phone no: 04132296356

E-mail: stevendkhar@ymail.com

DOI: 10.5530/jyp.2017.9.56

\section{INTRODUCTION}

Elevated levels of Low-Density Lipoprotein Cholesterol (LDL-C) is associated with an increased risk of coronary artery disease (CAD). ${ }^{1}$ LDL-C levels are affected by genetic and environmental factors. The basal risk in the form of genetic variations is not modifiable, although they are to a good extent modulated by environmental factors. Exploring and characterizing these genetic variations for each population would provide the background information needed to conduct further studies on lipid abnormalities and their treatment. Lipid traits are up to $60 \%$ heritable. $^{2}$ Indians being predisposed to $\mathrm{CAD},{ }^{3}$ it is essential to study the genetics of LDL-C to help predict CAD and to implement preventive measures in this high- risk population. Till now, 157 gene loci have been studied for association with blood lipid levels which could explain only $12-14 \%$ variation in the lipid levels. ${ }^{4}$

The clinical significance of genetic variants associated with lipid abnormalities depends on the frequency of genetic variants in the population of interest. Further, the normative data of frequency of single nucleotide polymorphism (SNP) is essential for planning and conducting genotypephenotype association studies. The frequency distribution of SNPs in other population cannot be used for planning studies in Indian population due to their unique genetic constitution. ${ }^{5}$

In earlier studies, the frequencies of genetic polymorphisms of genes involved in lipid and statin pathways such as $A p o A, A p o B,{ }^{6} \mathrm{CETP},{ }^{7}$ $\mathrm{ABCB} 1,{ }^{8,9}$ and $C Y P 3 A 5^{10}$ have been established among healthy South Indian Tamil population in our laboratory. For the current study, SNPs were selected based on their role in statin and cholesterol metabolism. These variants have been associated with altered LDL-C response to statins or associated with toxicity in other populations. However, there is no data among South Indian Tamil patients. Genes such as HMGCR, LDLR and CYP7A1 are involved in the cholesterol metabolic pathway. $H M G C R$ codes for 3-hydroxy-3-methylglutaryl-CoA reductase which is the rate-limiting enzyme for cholesterol synthesis. ${ }^{11} L D L R$ codes for low -density lipoprotein receptor, which is involved in endocytosis of LDL cholesterol. ${ }^{12}$ CYP7A1 belongs to Cytochrome 450 group of enzymes which catalyzes the rate limiting step in cholesterol catabolism to bile acids, the major route of cholesterol elimination from the body. ${ }^{13} A B C B 1$ encodes the membrane associated protein which is a member of the superfamily of ATP-binding cassette (ABC) transporters which is involved in efflux of statins. ${ }^{14}$ The SLCO1B1 codes for hepatic solute carrier organic anion transporter family member $1 \mathrm{~B} 1$ which is responsible for uptake of bilirubin and its metabolites and drugs like statins. ${ }^{15}$

Hence in the current study, we aimed to determine the frequencies of genetic variations for other genes in the lipid pathway such as $H M G C R$ (rs5908, rs17238540, rs12916), LDLR (rs688), CYP7A1 (rs3808607), $A B C B 1$ (rs1128503), and SLCO1B1 (rs4149056) in South Indian Tamil population. We also aimed to compare the studied genotype distributions with that of other ethnic populations. 


\section{MATERIALS AND METHODS}

The study was approved by the Institute Ethics Committee (Human Studies) of Jawaharlal Institute of Postgraduate Medical Education and Research (JIPMER), Puducherry, India. Written informed consent was obtained from participants recruited for the study. The study was conducted among 100 apparently healthy volunteers of either gender, aged between 27 and 70 years. All study subjects belonged to South Indian Tamil ethnicity which was defined as people with history of their past three generations residing in the state of Tamil Nadu or Puducherry and speaking Tamil language as the mother tongue.

Five milliliters of blood for genotyping was collected from the forearm of the patient in sitting posture, using ethylenediamine tetra-acetic acid (EDTA) as the anticoagulant. The cellular layer including the buffy coat was separated using ultracentrifugation at 2500 rotations per minute (RPM) for $10 \mathrm{~min}$, and stored at $-20^{\circ} \mathrm{C}$, until extraction. DNA extraction was performed using standard phenol chloroform method. Genotyping was done with allelic discrimination assays, with the kits obtained from Applied BioSystems (ABI) USA. The assay kits were based on TaqMan Technology. Real-Time PCR platform ABI 7300 was used. The allelic call was read with the help of sequence manipulation suite (SMS) version 1.4. The genotyping results were confirmed by running the samples in triplicates (representative genotypes) and by Sanger sequencing.

Hardy-Weinberg equilibrium for the studied genotypes was tested using Chi-square test. Genotype frequencies from the current study were compared with data from other studies using Fisher's Exact Test or Chisquare test. $\mathrm{P}$ value less than 0.05 was considered significant. All statistical tests were done using GraphPad Instat v 3.0 (GraphPad Software Inc., San Diego, CA, USA).

\section{RESULTS}

The study subjects included 75 females and 25 males with a mean age of $45.5 \pm 10.9$ (SD). The genotype and allele frequencies established in this study are given in Table 1. HMGCR rs5908 was found to be very rare in the study population with only one subject having a heterozygous variant genotype with the absence of homozygous variant genotype. HMGCR rs17238540 polymorphism was also less prevalent in our population with the frequency of 3.5\%.The variant alleles of HMGCR rs12916, CYP7A1 rs3808607 and ABCB1 rs1128503 polymorphisms were found to be more prevalent. The variant genotype of SLCO1B1 rs4149056 polymorphism was not found in our population. All genotypes except $L D L R$ rs688 polymorphism did not show significant deviation from Hardy -Weinberg equilibrium.

Data of genotype and allele counts of the studied SNPs from North In$\mathrm{dia}^{16}$ and other major ethnic groups retrieved from 1000 genomes project phase 3 population ${ }^{17}$ are given in Table 2 . The distribution of variant genotypes of HMGCR rs5908 observed in our population was significantly different only from North Indian population. However, distribution of HMGCR rs17238540 genotypes were significantly different from other populations except for CEU (Utah residents with Northern and Western European Ancestry), MXL (Mexican Ancestry in Los-Angeles, California), and Europeans from Norfolk-United Kingdom. ${ }^{18}$ The distribution of HMGCR rs12916 genotypes was significantly different from that of CEU, YRI (Yoruba in Ibadan, Nigeria) and MXL population. The distribution of $L D L R$ rs688 genotypes in the current study was distinctly different from the other populations except that of CHB (Han Chinese in Beijing, China). The distribution of genotypes of CYP7A1 rs3808607 polymorphism was significantly different from a north Indian study which included patients from Punjab, Haryana and Chandigarh and also from YRI and JPT (Japanese in Tokyo, Japan)but not from other population of North India. ${ }^{19}$ The distribution of $A B C B 1$ rs1128503 was significantly different from that of CEU and YRI population. The distribution of genotypes of SLCO1B1 in our population was significantly different from that of GIH (Gujarati Indian in Houston), CEU, CHB, and YRI.

Table 1: Genotype and allelic frequencies of the studied genetic polymorphisms

\begin{tabular}{|c|c|c|c|c|c|c|c|}
\hline \multirow[t]{3}{*}{ Gene } & SNP & \multicolumn{3}{|c|}{ Genotype (\%) } & \multicolumn{2}{|c|}{ Allele (\%) } & \multirow[t]{2}{*}{$\begin{array}{c}\text { HWE } \\
\text { p-value }\end{array}$} \\
\hline & $1912 A>C(\operatorname{cc5} 5008)$ & AA & AG & GG & A & G & \\
\hline & (1) & 99 & 1 & 0 & 99.5 & 0.5 & 0.95 \\
\hline \multirow{4}{*}{ HMGCR } & & TT & TG & GG & $\mathrm{T}$ & G & \\
\hline & $1 / 403248018(151 / 250340)$ & 93 & 7 & 0 & 96.5 & 3.5 & 0.71 \\
\hline & $* 272 C>\mathrm{T}(\mathrm{rc} 12016)$ & $\mathrm{CC}$ & CT & TT & $\mathrm{C}$ & $\mathrm{T}$ & \\
\hline & & 36 & 46 & 18 & 59 & 41 & 0.62 \\
\hline \multirow{2}{*}{$L D L R$} & $1773 C>\mathrm{T}(\mathrm{rc688})$ & $\mathrm{CC}$ & CT & TT & C & $\mathbf{T}$ & \\
\hline & & 64 & 20 & 16 & 74 & 26 & $<0.0001^{\star}$ \\
\hline \multirow{2}{*}{ CYP7A1 } & $58500365 \mathrm{G}>\mathrm{T}(\mathrm{rc} 3808607)$ & GG & GT & TT & G & $\mathrm{T}$ & \\
\hline & & 22 & 40 & 38 & 42 & 58 & 0.07 \\
\hline \multirow{2}{*}{$A B C B 1$} & $87550285 \mathrm{~A}>\mathrm{G}(\mathrm{rc} 1128503)$ & GG & AG & AA & G & A & \\
\hline & (1) & 18 & 47 & 35 & 41.5 & 58.5 & 0.74 \\
\hline \multirow{2}{*}{ SLCO1B1 } & $521 \mathrm{~T}>C(\mathrm{rs} 4149056)$ & TT & CT & CC & $\mathbf{T}$ & C & \\
\hline & & 86 & 14 & 0 & 93 & 7 & 0.45 \\
\hline
\end{tabular}

HWE - Hardy Weinberg Equilibrium p value.

${ }^{*} \mathrm{p}$ value less than 0.05 , significant deviation from Hardy Weinberg Equilibrium 
Table 2: Comparison of genotype counts of Tamilian population with other populations

\begin{tabular}{|c|c|c|c|c|c|c|c|c|}
\hline SNP & Population & $\mathrm{N}$ & \multicolumn{3}{|c|}{ Genotype count } & \multicolumn{2}{|c|}{ Allele count } & $P$ value \\
\hline \multirow{2}{*}{ HMGCR rs5908 } & \multirow{2}{*}{ Tamilians } & \multirow{2}{*}{100} & AA & AG & GG & A & G & \\
\hline & & & 99 & $(1$ & 0) & 199 & 1 & \\
\hline \multirow{25}{*}{$\begin{array}{c}\text { HMGCR } \\
\text { rs17238540 }\end{array}$} & North Indians[16] & 150 & 126 & $(22$ & 2) & 274 & 26 & $<0.0001^{*}$ \\
\hline & GIH[17] & 103 & 103 & 0 & 0 & 206 & 0 & 0.49 \\
\hline & ITU\# & 102 & 102 & 0 & 0 & 204 & 0 & 0.49 \\
\hline & BEB\# & 86 & 85 & $(1$ & $0)$ & 171 & 1 & 1.00 \\
\hline & PJL\# & 96 & 93 & $(3$ & $0)$ & 189 & 3 & 0.36 \\
\hline & STU\# & 102 & 100 & $(2$ & 0) & 202 & 2 & 1.00 \\
\hline & CEU\# & 99 & 94 & $(5$ & $0)$ & 193 & 5 & 0.12 \\
\hline & $\mathrm{CHB} \#$ & 103 & 103 & 0 & 0 & 206 & 0 & 0.49 \\
\hline & YRI\# & 108 & 108 & 0 & 0 & 216 & 0 & 0.48 \\
\hline & JPT\# & 104 & 104 & 0 & 0 & 208 & 0 & 0.49 \\
\hline & MXL\# & 64 & 63 & $(1$ & $0)$ & 127 & 1 & 1.00 \\
\hline & \multirow{2}{*}{ Tamilians } & \multirow{2}{*}{100} & TT & GT & GG & $\mathbf{T}$ & G & \\
\hline & & & 93 & $(7$ & 0) & 193 & 7 & \\
\hline & North Indians & 150 & 150 & 0 & 0 & 300 & 0 & $0.001^{*}$ \\
\hline & $\mathrm{GIH}$ & 103 & 103 & 0 & 0 & 206 & 0 & $0.006^{*}$ \\
\hline & ITU & 102 & 102 & 0 & 0 & 204 & 0 & $0.006^{*}$ \\
\hline & $\mathrm{BEB}$ & 86 & 86 & 0 & 0 & 172 & 0 & $0.01^{*}$ \\
\hline & PJL & 96 & 96 & 0 & 0 & 192 & 0 & $0.01^{*}$ \\
\hline & STU & 102 & 101 & $(1$ & 0) & 203 & 1 & $0.03^{*}$ \\
\hline & $\mathrm{CEU}$ & 99 & 96 & $(3$ & 0) & 195 & 3 & 0.33 \\
\hline & CHB & 103 & 103 & 0 & 0 & 206 & 0 & $0.006^{*}$ \\
\hline & YRI & 108 & 88 & $(19$ & 1) & 195 & 21 & $0.02^{*}$ \\
\hline & JPT & 104 & 104 & 0 & 0 & 208 & 0 & $0.006^{*}$ \\
\hline & MXL & 64 & 59 & $(5$ & 0) & 123 & 5 & 1.00 \\
\hline & European[18] & 23011 & 22010 & (989 & 12) & 45009 & 1013 & 0.19 \\
\hline \multirow{13}{*}{ HMGCR rs 12916} & \multirow{2}{*}{ Tamilians } & \multirow{2}{*}{100} & CC & $\mathbf{C T}$ & TT & C & $\mathbf{T}$ & \\
\hline & & & 36 & 46 & 18 & 118 & 82 & \\
\hline & North Indians & 150 & 64 & 63 & 23 & 191 & 109 & 0.56 \\
\hline & GIH & 103 & 35 & 55 & 13 & 125 & 81 & 0.45 \\
\hline & ITU & 102 & 33 & 39 & 30 & 105 & 99 & 0.15 \\
\hline & BEB & 86 & 31 & 37 & 18 & 99 & 73 & 0.86 \\
\hline & PJL & 96 & 25 & 52 & 19 & 102 & 90 & 0.31 \\
\hline & STU & 102 & 33 & 49 & 20 & 115 & 89 & 0.85 \\
\hline & $\mathrm{CEU}$ & 99 & 17 & 46 & 36 & 80 & 118 & $0.001^{*}$ \\
\hline & $\mathrm{CHB}$ & 103 & 29 & 49 & 25 & 107 & 99 & 0.37 \\
\hline & YRI & 108 & 7 & 28 & 73 & 42 & 174 & $<0.0001^{*}$ \\
\hline & JPT & 104 & 26 & 58 & 20 & 110 & 98 & 0.22 \\
\hline & MXL & 64 & 12 & 24 & 28 & 48 & 80 & $0.001^{*}$ \\
\hline \multirow{6}{*}{ LDLR rs688 } & \multirow{2}{*}{ Tamilians } & \multirow{2}{*}{100} & CC & $\mathbf{C T}$ & TT & C & $\mathbf{T}$ & \\
\hline & & & 64 & 20 & 16 & 148 & 52 & \\
\hline & $\mathrm{GIH}$ & 103 & 42 & 45 & 16 & 129 & 77 & $0.0009^{*}$ \\
\hline & ITU & 102 & 35 & 52 & 15 & 122 & 82 & $<0.0001^{*}$ \\
\hline & BEB & 86 & 38 & 36 & 12 & 112 & 60 & $0.004^{*}$ \\
\hline & PJL & 96 & 36 & 40 & 20 & 112 & 80 & $0.0006^{*}$ \\
\hline
\end{tabular}


Table 2: Comparison of genotype counts of Tamilian population with other populations

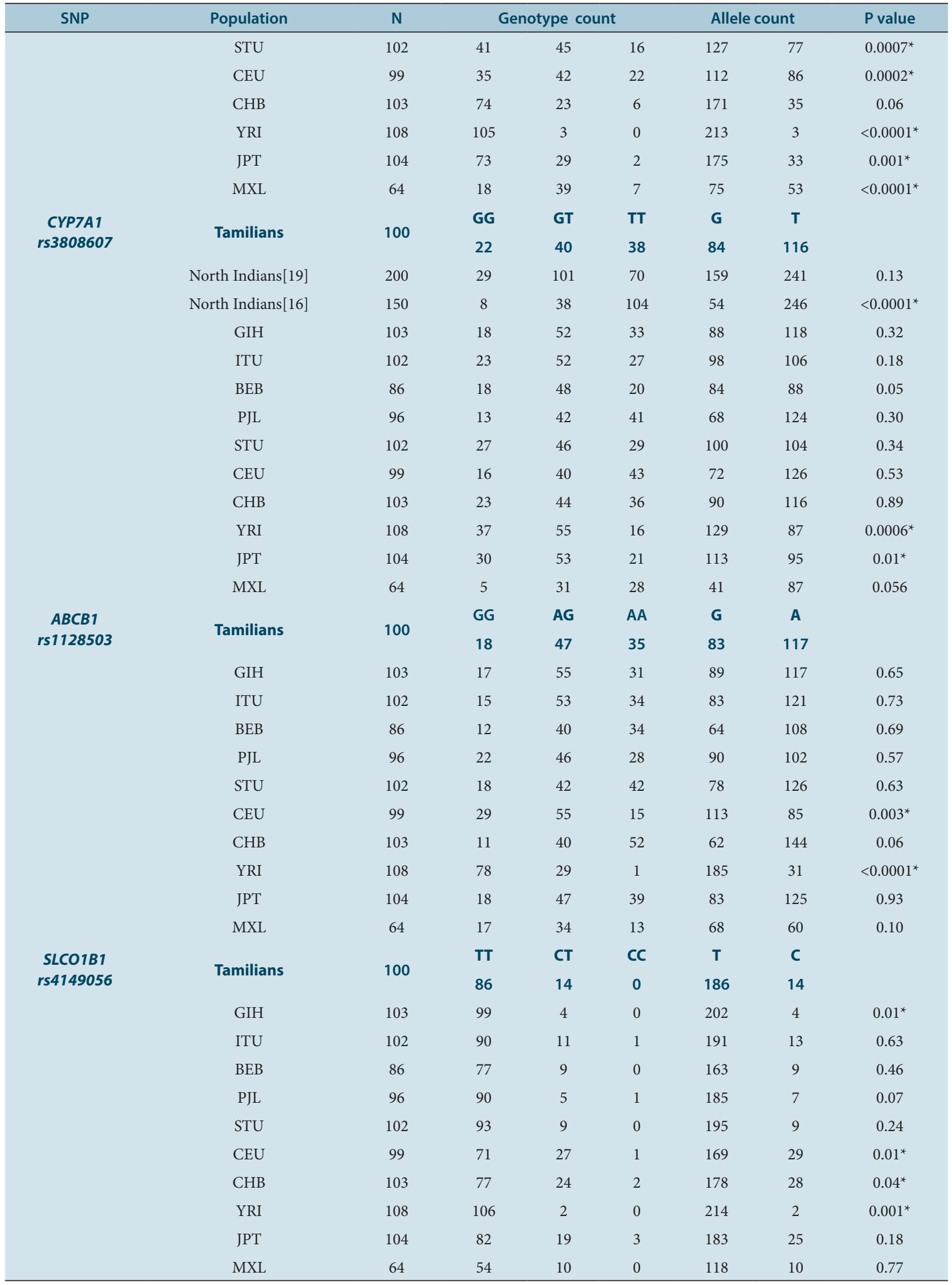

\#Data obtained from 1000 genome project; GIH- Gujarati Indian in Houston, TX, ITU-Indian Telugu in the UK, BEB-Bengali in Bangladesh, PJL-Punjabi in Lahore, Pakistan, STU-Sri Lankan Tamil in the UK, CEU-Utah residents with Northern and Western European Ancestry, CHBHan Chinese in Beijing, China, YRI-Yoruba in Ibadan, Nigeria, JPT-Japanese in Tokyo Japan, MXL-Mexican Ancestry in Los-Angeles, California. Parentheses ( ) indicates genotypes were combined for analysis, due to low numbers. Genotype count from current study were compared with data from other studies using Fisher's Exact Test or Chi square test. 


\section{DISCUSSION}

The present study has determined the normative data on allele and genotype frequencies for SNPs of five genes namely, HMGCR (rs5908, rs17238540, rs12916), LDLR (rs688), CYP7A1 (rs3808607), ABCB1 (rs1128503), and SLCO1B1 (rs4149056). These genes are involved in the maintenance of plasma lipid levels and the metabolism of statin drugs. To the best of our knowledge this study is the first to describe this information for South Indian Tamil population. The normative data on allele and genotype frequencies are important for planning of any genetic study to estimate sample size and to understand the clinical implications of a genetic variation. Thus, the current study provides the background information needed for future studies on pharmacogenetics of lipid metabolism and statins among South Indian Tamil population.

Genetic variants of HMG-CoA reductase (HMGCR) have been widely studied for their effect on lipid levels and variations in therapeutic responses to different statins. HMGCR variants have been associated with diminished response to pravastatin and simvastatin but not to fluvastatin $^{20}$ and atorvastatin. ${ }^{21}$ Further, they were also found to be associated with varied response between different ethnic groups. ${ }^{22}$ Among the three SNPs studied in HMGCR gene, variant genotypes of rs12916 and rs17238540 are more common in the study population. In contrast, $H M G C R$ rs5908 variant being in low frequency may have less clinical relevance for the study population, even if functionally significant. Functional studies on rs12916 and rs17238540 variants may have clinical relevance in elucidating the effect of these SNPs on lipid homeostasis.

The HMGCR rs17238540 was found to be associated with reduced efficacy of statins in terms of lowering plasma levels of total cholesterol (TC) and LDL-C. Individuals with heterozygous variant genotype (GT) had $19 \%$ lower reduction in LDL-C when treated with pravastatin. ${ }^{23}$ In the GoDARTS study, individuals with HMGCR rs17238540 variant G allele were associated with failure to achieve lipid lowering target. ${ }^{24}$ In a study by Poduri et al., in North Indian population, the variant genotypes of HMGCR rs17238540 was associated with significantly higher LDL-C levels after atorvastatin therapy compared to wild type. ${ }^{25}$ However, in a study by Thompson et al., this SNP was not associated with response to atorvastatin. ${ }^{21}$ In few studies, HMGCR rs17238540 was not associated with baseline lipid values. ${ }^{18,23,24}$ In the multi-ethnic study of atherosclerosis the HMGCR haplotypes, ${ }^{26}$ and these genotypes among North Indians were associated with baseline lipid levels respectively. ${ }^{25}$ It was also observed that the HMGCR rs 17238540 might negate some of the observed pleiotropic effects of statins, such as improved endothelial function, decreased platelet aggregability and reduced vascular inflammation and was also found to be associated with stroke risk in the EPICNorfolk study. ${ }^{27}$

The HMGCR rs12916 locus has been associated with total cholesterol and LDL-C levels among Europeans as well as East Asians and South Asians in a genome wide study. ${ }^{28}$ In candidate gene studies the mean percentage reduction in LDL-C with HMGCR rs 12916 polymorphisms among Chinese were found to be highest for homozygous variant, least for wild genotype and intermediate for heterozygous individuals after treatment with statins ${ }^{29}$ while, studies among North Indians revealed that variant genotypes of this polymorphisms were responsible for poor response to atorvastatin in terms of LDL-C lowering. ${ }^{25}$ Given the high prevalence of this genetic polymorphism among Tamilians, it would be of interest to determine the effect of this polymorphism on the lipid levels in our population.

LDL receptor gene (LDLR) is a commonly studied genetic locus for dyslipidemias. Genetic variations in LDL receptor gene may greatly reduce or abolish the function of LDL receptor. This may lead to increase in circulating LDL-C levels and risk of CAD. ${ }^{30}$ Although LDLR gene mutations are associated with familial hypercholesterolemia (FH), less dysfunctional variants have been associated with response to statin in non -FH individuals. ${ }^{31}$ Studies by Haiyan Zhu and his colleagues have identified SNP LDLR rs688 to be present in $60 \%$ of Caucasians and associated with a significant $10 \%$ increase in total and LDL-cholesterol in pre-menopausal women. ${ }^{32}$ This SNP has been recently shown to alter splicing efficiency, with the $\mathrm{T}$ allele being associated with increased total and LDL-cholesterol levels among premenopausal women. ${ }^{33}$ Since this SNP is present in high frequency in the study population, and since it has not been studied among Indian population at large, more studies on the functional significance, of this SNP might yield useful information on the genetics of LDL-C in our population.

The genotype distribution of CYP7A1 rs3808607 variants among Tamilian population was found to be similar to another study involving people from Lucknow region of North India, ${ }^{19}$ whereas it was significantly different from the results obtained in a study among North Indian regions of Punjab, Haryana, and Chandigarh, ${ }^{16}$ revealing the diversity even among North Indians. The genetic variants of CYP7A1 rs3808607, $A B C B 1$ rs1128503, and SLCO1B1 rs4149056, although similar to most other populations in frequency distribution, were found to occur at a higher frequency in the study population and may have clinical relevance if functional significance can be demonstrated in future studies.

\section{CONCLUSION}

The study has established the allele and genotype frequency distributions for HMGCR (rs5908, rs17238540, rs12916), LDLR (rs688), CYP7A1 (rs3808607), ABCB1 (rs1128503), and SLCO1B1 (rs4149056) genetic variants among healthy subjects of South Indian Tamil population. The South Indian Tamil population is a unique ethnic group in terms of genetic polymorphisms in the cholesterol and statin metabolic pathways.

\section{Funding}

This study was supported by the financial aid from JIPMER intramural fund for the start-up research and was completed with the financial support by Department of Biotechnology - Project San No-BT/PR5130/ MED/12/553/2012.

\section{ACKNOWLEDGEMENT}

The author wishes to thank Dr. Anusha N, Senior Resident, Department of Pharmacology, JIPMER for her help with the initial draft of the document.

\section{CONFLICTS OF INTEREST}

There are no conflicts of interest.

\section{ABBREVIATION USED}

ApoA: Apolipoprotein A; ApoB: Apolipoprotein B; CETP: Cholesteryl ester transfer protein; $\boldsymbol{A B C B 1 :}$ ATP binding Cassette subfamily B member 1; CYP3A5: Cytochrome P450 family 3 subfamily A member 5; CYP7A1: Cytochrome P450 family 7 subfamily A member 1.

\section{REFERENCES}

1. National Cholesterol Education Program (NCEP) Expert Panel on Detection, Evaluation, and Treatment of High Blood Cholesterol in Adults (Adult Treatment Panel III). Third Report of the National Cholesterol Education Program (NCEP) Expert Panel on Detection, Evaluation, and Treatment of High Blood Cholesterol in Adults (Adult Treatment Panel III) final report. Circulation. 2002;106(25):3143421.

2. Tada H, Won H-H, Melander O, Yang J, Peloso GM, Kathiresan S. Multiple associated variants increase the heritability explained for plasma lipids and coronary artery disease. Circ Cardiovasc Genet. 2014;7(5):583-7.

3. Enas EA, Senthilkumar A. Coronary Artery Disease in Asian Indians: An Update 
And Review. The Internet Journal of Cardiology [Internet]. 2001 Dec 31 [cited 2017 Jan 11]; 1(2). Available from: http://ispub.com/IJC/1/2/4493.

4. Justesen JM, Allin KH, Sandholt CH, Borglykke A, Krarup NT, Grarup N, et al. Interactions of Lipid Genetic Risk Scores With Estimates of Metabolic Health in a Danish Population. Circ Cardiovasc Genet. 2015;8(3):465-72.

5. Reich D, Thangaraj K, Patterson N, Price AL, Singh L. Reconstructing Indian population history. Nature. 2009;461 (7263):489-94. doi:10.1038/nature08365.

6. Padmaja N, Ravindra KM, Adithan C. Apolipoprotein Al and Apolipoprotein B gene polymorphisms and lipid profile in Tamilian population. Ann Hum Biol. 2009;36(2):220-7

7. Padmaja N, Ravindra KM, Soya SS, Adithan C. Common variants of Cholesteryl ester transfer protein gene and their association with lipid parameters in healthy volunteers of Tamilian population. Clinica Chimica Acta. 2007;375(1-2):140-6.

8. Ramasamy K, Sisy Sam S, Chandrasekaran A. Allele and genotype frequency of MDR1 C3435T in Tamilian population. Drug Metab Pharmacokinet. 2006;21(6):506-8

9. Umamaheswaran G, Krishna KD, Kayathiri D, Rajan S, Shewade DG, Dkhar SA, et al. Inter and intra-ethnic differences in the distribution of the molecular variants of TPMT, UGT1A1 and MDR1 genes in the South Indian population. Mol Biol Rep. 2012 May;39(5):6343-51.

10. Krishnakumar D, Gurusamy U, Dhandapani K, Surendiran A, Baghel R, Kukreti $\mathrm{R}$, et al. Genetic polymorphisms of drug-metabolizing phase I enzymes CYP2E1, CYP2A6 and CYP3A5 in South Indian population. Fundam Clin Pharmacol. 2012;26(2):295-06

11. HMGCR Gene - GeneCards | HMDH Protein | HMDH Antibody [Internet]. [cited 2016 Oct 25]. Available from: http://www.genecards.org/cgi-bin/carddisp. pl?gene=HMGCR .

12. LDLR Gene - GeneCards | LDLR Protein | LDLR Antibody [Internet]. [cited 2016 Oct 25]. Available from: http://www.genecards.org/cgi-bin/carddisp. pl?gene=ldlr.

13. CYP7A1 Gene - GeneCards | CP7A1 Protein | CP7A1 Antibody [Internet]. [cited 2016 Oct 23]. Available from: http://www.genecards.org/cgi-bin/carddisp. pl?gene $=$ CYP7A 1 .

14. ABCB1 Gene - GeneCards | MDR1 Protein | MDR1 Antibody [Internet]. [cited 2016 Oct 25].Availablefrom:http://www.genecards.org/cgi bin/carddisp. pl?gene $=A B C B 1 \&$ keywords $=$ ABCB1.

15. SLCO1B1 Gene - GeneCards | SO1B1 Protein | SO1B1 Antibody [Internet]. [cited 2016 Oct 23].Availablefrom:http://www.genecards.org/cgi-bin/carddisp.pl ?gene $=$ SLCO1B1\&keywords $=$ SLCO1B1.

16. Poduri A, Khullar M, Bahl A, Sharma Y, Talwar K. A Combination of Proatherogenic Single-Nucleotide Polymorphisms Is Associated with Increased Risk of Coronary Artery Disease and Myocardial Infarction in Asian Indians. DNA and Cell Biology. 2009;28(9):451-60.

17. A global reference for human genetic variation, The 1000 Genomes Project Consortium, Nature.2015 Oct; 526: 68-74. doi:10.1038/nature15393.

18. Freitas RN, Khaw K-T, Wu K, Bowman R, Jeffery $H$, Luben $\mathrm{R}$, et al. A single nucleotide polymorphism in the 3-hydroxy-3-methylglutaryl-coenzyme $A$ reductase gene (HMGCR) influences the serum triacylglycerol relationship with dietary fat and fibre in the European Prospective Investigation into Cancer and Nutrition in Norfolk (EPIC-Norfolk) study. British Journal of Nutrition. 2010;104(5):765-72.
19. Srivastava A, Pandey S, Choudhuri G, Mittal B. Role of genetic variant A-204C of cholesterol 7 $\alpha$-hydroxylase (CYP7A1) in susceptibility to gallbladder cancer. Molecular Genetics and Metabolism. 2008;94(1):83-9.

20. Hu M, Mak WWL, Chu TTW, Waye MMY, Tomlinson B. Pharmacogenetics of HMG-CoA Reductase Inhibitors: Optimizing the Prevention of Coronary Heart Disease. Current Pharmacogenomics and Personalized Medicine. 2009;7(1):126.

21. Thompson JF, Man M, Johnson KJ, Wood LS, Lira ME, Lloyd DB, et al. An association study of 43 SNPs in 16 candidate genes with atorvastatin response. Pharmacogenomics J. 2005;5:352-8.

22. Krauss RM, Mangravite LM, Smith JD, Medina MW, Wang D, Guo X, et al. Variation in the 3-Hydroxyl-3-Methylglutaryl Coenzyme A Reductase Gene Is Associated With Racial Differences in Low-Density Lipoprotein Cholesterol Response to Simvastatin Treatment. Circulation. 2008;117(12):1537-44.

23. Chasman DI, Posada D, Subrahmanyan L, Cook NR, Stanton VP, Ridker PM. Pharmacogenetic Study of Statin Therapy and Cholesterol Reduction. JAMA: The Journal of the American Medical Association. 2004;291(23):2821 -7.

24. Donnelly LA, Doney AS, Dannfald J, Whitley AL, Lang CC, Morris AD, et al. A paucimorphic variant in the HMG-CoA reductase gene is associated with lipidlowering response to statin treatment in diabetes: a GoDARTS study. Pharmacogenet. Genomics. 2008;18:1021-6.

25. Poduri A, Khullar M, Bahl A, Sehrawat B s. Sharma Y Talwar KK. Common Variants of HMGCR, CETP, APOAI, ABCB1, CYP3A4, and CYP7A1 Genes as Predictors of Lipid-Lowering Response to Atorvastatin Therapy. DNA and Cell Biology. 2010;29(10):629-37.

26. Chen Y-C, Chen Y-DI, Li X, Post W, Herrington D, Polak JF, et al. The HMG-CoA reductase gene and lipid and lipoprotein levels: the multi-ethnic study of atherosclerosis. Lipids. 2009;44(8):733-43.

27. Freitas RN, Khaw K-T, Wu K, Bowman R, Jeffery H, Luben R, et al. HMGCR gene polymorphism is associated with stroke risk in the EPIC-Norfolk study. Eur J Cardiovasc Prev Rehabil. 2010;17(1):89-93.

28. Teslovich TM, Musunuru K, Smith AV, Edmondson AC, Stylianou IM, Koseki M, et al. Biological, Clinical, and Population Relevance of 95 Loci for Blood Lipids. Nature. 2010;466(7307):707-13.

29. Chien K-L, Wang K-C, Chen Y-C, Chao C-L, Hsu H-C, Chen M-F, et al. Common sequence variants in pharmacodynamic and pharmacokinetic pathway-related genes conferring LDL cholesterol response to statins. Pharmacogenomics. 2010;11(3):309-17.

30. Cambien F, Tiret L. Genetics of Cardiovascular Diseases. Circulation. 2007;116(15):1714-24

31. Mangravite LM, Thorn CF, Krauss RM. Clinical implications of pharmacogenomics of statin treatment. Pharmacogenomics J. 2006;6(6):360-74.

32. Zhu H, Tucker HM, Grear KE, Simpson JF, Manning AK, Cupples LA, et al. A Common Polymorphism Decreases Low-Density Lipoprotein Receptor Exon 12 Splicing Efficiency and Associates with Increased Cholesterol. Hum Mol Genet. 2007;16(14):1765-72.

33. Martinelli N, Girelli D, Lunghi B, Pinotti M, Marchetti G, Malerba G, et al. Polymorphisms at LDLR locus may be associated with coronary artery disease through modulation of coagulation factor VIII activity and independently from lipid profile. Blood. 2010;116(25):5688-97.

Article History: Submission Date: 04-02-17; Received Date: 11-02-17; Acceptance Date: 18-02-17.

Cite this article: Indumathi C, Kumar ASA, Surendiran A, Dkhar SA. Frequencies of Genetic Polymorphisms of the Cholesterol and Statin Metabolic Pathway Genes among Healthy South Indian Tamil Population. J Young Pharm. 2017;9(2):284-9. 